(c) (c) 2015 IEEE. Personal use of this material is permitted. Permission from IEEE must be obtained for all other uses, in any current or future media, including reprinting/republishing this material for advertising or promotional purposes, creating new collective works, for resale or redistribution to servers or lists, or reuse of any copyrighted component of this work in other works.

\title{
Performance Limit of Two-Pump Brillouin Fiber Sensors Obtained by Manakov Modulation Instability
}

\author{
Mehdi Alem*, Marcelo A. Soto, and Luc Thévenaz \\ Group for Fiber Optics, Institute of Electrical Engineering \\ EPFL Swiss Federal Institute of Technology \\ SCI STI LT, Station 11, 1015 Lausanne, Switzerland \\ "E-mail: mehdi.alem@epfl.ch
}

\begin{abstract}
The Manakov modulation instability is shown to determine the performance limit of long-distance Brillouin fiber sensors utilizing orthogonally-polarized pumps. Numerical simulations of the Manakov equation are supported by the experimental measurement of a $25-\mathrm{km}$ Brillouin fiber sensor to verify the model. The study reveals that the use of orthogonallypolarized pulses decreases the pump depletion due to modulation instability leading to an enhancement of the sensing distance.
\end{abstract}

Keywords-Kerr effect; modulation instability; distributed fiber sensor; Manakov system; stimulated Brillouin scattering

\section{INTRODUCTION}

There has been an extensive effort during the last decade to increase the sensing range achieved by Brillouin optical time domain analysis (BOTDA) distributed fiber sensors [1]. Reaching long sensing distances requires increasing pump and probe powers launched into the sensing fiber to provide a high enough signal-to-noise ratio (SNR) at the end of the fiber. However, using a high peak-power pump excites detrimental nonlinear effects like modulation instability (MI) and stimulated Raman scattering (SRS), which deplete the pump and decrease the maximum sensing distance of the system [2]. Compared to SRS, MI shows a lower critical power and thus determines the maximum sensing range of a BOTDA sensor [2]. The effect of MI on single-pump distributed sensors has been studied in [2] and [3], while in [4] the maximum range has been derived based on the depletion length induced by MI. Using multi-wavelength pumps in parallel [5] and orthogonal [6] polarizations has been proposed recently to improve the BOTDA performance. In this paper, we show that while in the parallel pumps case the system is highly affected by four-wave mixing (FWM), the main limitation in the orthogonal case is determined by the vector modulation instability.

\section{MANAKOV MODULATION INSTABILITY}

Modulation instability in optical fibers is the result of an interaction between the group velocity dispersion (GVD) in anomalous regime $\left(\beta_{2}<0\right)$ and the nonlinear Kerr effect represented by the nonlinear coefficient $\gamma$. In the time domain, MI breaks a $\mathrm{CW}$ light into a train of soliton-like pulses, while in the frequency domain it produces symmetric sidebands around the pump wavelength. Standard single-mode fibers (SMFs), mostly used in distributed sensing, have anomalous dispersion at $1550 \mathrm{~nm}$ and thus suffer from modulation instability. In the case of a single pump, the pump pulse

\author{
Javier Urricelqui ${ }^{\dagger}$, Mikel Sagues, and Alayn Loayssa \\ Department of Electrical and Electronic Engineering \\ Universidad Pública de Navarra \\ Campus Arrosadia s/n, 31006 Pamplona, Spain \\ 'E-mail: javier.urricelqui@unavarra.es
}

envelope $A(z, t)$ evolves along the fiber according to the scalar nonlinear Schrödinger equation (NLSE) as follows [7]:

$$
\frac{\partial A}{\partial z}+i \frac{\beta_{2}}{2} \frac{\partial^{2} A}{\partial t^{2}}+\frac{\alpha}{2} A=i \gamma|A|^{2} A,
$$

where $\alpha$ is the fiber attenuation. Scalar modulation instability in standard BOTDA has been studied thoroughly by analyzing (1) and the model has been verified experimentally [4]. Considering orthogonally polarized pumps one needs to extend the scalar analysis of MI to its vector counterpart where the vector NLSE governs the polarization components of the lightwave [7]. The form of the vector NLSE depends on the birefringence characteristics of the fiber. Any deviation from perfectly cylindrical structure of optical fibers due to oval cross-section or applied stress can induce an anisotropy that leads to birefringence. This anisotropy breaks the degeneracy of the two orthogonally polarized modes of the hybrid mode $\mathrm{HE}_{11}$ and thus the fiber acts like a two-mode fiber. SMFs are categorized as 'randomly varying birefringent' fibers in which birefringence changes randomly over a length scale $\sim 10 \mathrm{~m}$ in both amplitude and axis [8]. These random fluctuations force the Stokes vector to sweep the entire surface of the Poincaré sphere over a length scale $\sim 1 \mathrm{~km}$. For fibers of a few kilometers long, averaging over the entire Poincaré sphere leads to the following coupled nonlinear Schrödinger equations referred to as the Manakov system [7]-[9]:

$$
\left\{\begin{array}{l}
\frac{\partial u}{\partial z}+i \frac{\beta_{2}}{2} \frac{\partial^{2} u}{\partial t^{2}}+\frac{\alpha}{2} u=i \gamma\left(|u|^{2}+|v|^{2}\right) u \\
\frac{\partial v}{\partial z}+i \frac{\beta_{2}}{2} \frac{\partial^{2} v}{\partial t^{2}}+\frac{\alpha}{2} v=i \gamma\left(|u|^{2}+|v|^{2}\right) v
\end{array},\right.
$$

where $u$ and $v$ are two orthogonal polarization components.

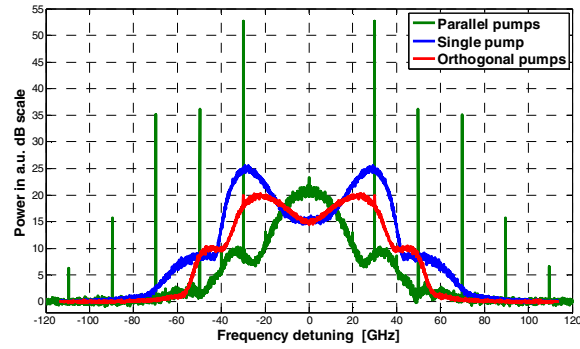

Fig. 1. MI gain spectrum for a $10 \mathrm{~km}$ SMF with $\alpha=0.2 \mathrm{~dB} / \mathrm{km}$, $\beta_{2}=-22 \mathrm{ps}^{2} / \mathrm{km}$ and $\gamma=1.8 \mathrm{~W}^{-1} / \mathrm{km}$ in three pump scenarios: single pump, two parallel and two orthogonal pumps with a frequency spacing of $20 \mathrm{GHz}$. 
It is worth mentioning that in the Manakov system, as it is clear from (2), the self-phase modulation (SPM) and crossphase modulation (XPM) of the orthogonal components have equal effects on each other. Here (1) and (2) have been solved numerically using the split-step Fourier method [7] and in order to consider the background noise as a seeding to MI, a Monte Carlo simulation has been performed. Fig. 1 shows the MI gain spectrum obtained by simulating (1) and (2) for three scenarios: single pump with $250 \mathrm{~mW}$ peak power, parallel pumps with $125 \mathrm{~mW}$ each, and orthogonal pumps of $125 \mathrm{~mW}$ each. Results point out that in the parallel pumps case FWM is the most relevant effect, while with orthogonal pumps FWM is highly suppressed [9], making the Manakov MI the dominant effect. Interestingly, the Manakov MI gain is lower than the scalar MI, imposing less restrictive limitations to the system.

\section{EXPERIMENTAL MEASUREMENTS}

Fig. 2 depicts the concept of the experimental setup. Two pulsed pumps with frequency spacing $19.1 \mathrm{GHz}$ are generated using a Mach-Zehnder electro-optic modulator [6]. Their states of polarization are controlled by a polarization controller, a differential group delay module and a programmable optical filter to have the three different scenarios: single pump, double parallel pumps and double orthogonal pumps. An electro-optic phase modulator driven by a $1.3 \mathrm{GHz}$ RF signal generates the probe wave (carrier and two sidebands). Since the Brillouin frequency of the fiber is $10.85 \mathrm{GHz}$, the low-frequency pump induces Brillouin loss on the upper probe sideband, while the high-frequency pump induces Brillouin gain on the lower probe sideband. The probe components after propagation along the fiber are directed into a photo-detector and the resulting signal is demodulated and then captured by an oscilloscope.

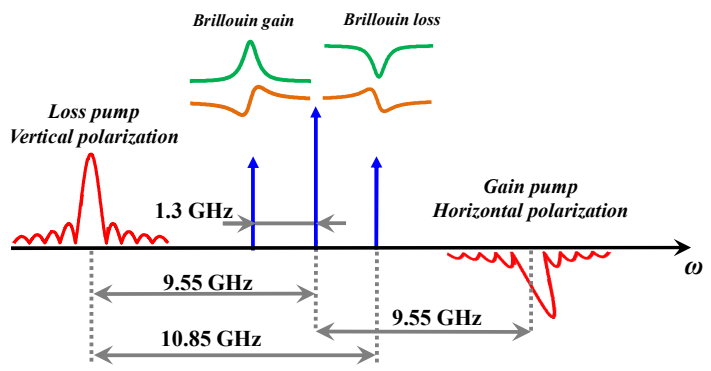

Fig. 2. Brillouin interactions of probes and orthogonally-polarized pumps.

Fig. 3 shows the power evolution along the fiber for the three scenarios: single pump, parallel pumps and orthogonal pumps obtained by measurement and simulation. As it is evident from the figure, the numerical simulations of (1) and (2) follows accurately the experimental results demonstrating the validity of the model. Fig. 3(a) compares the single-pump BOTDA with the double parallel pumps. This clearly shows more than $50 \%$ pump depletion at the end of the fiber in both scenarios when compared to the undepleted exponential decay. The deep power oscillation for the parallel pumps at $5 \mathrm{~km}$ distance confirms that the FWM between parallel pumps dominates the sensor response. Fig. 3(b) reveals the reduction of MI-induced pump depletion obtained with orthogonal pumps compared to a single pump. This actually leads to power evolution close to the ideal undepleted case when using orthogonal pumps, providing higher SNR at the fiber end.
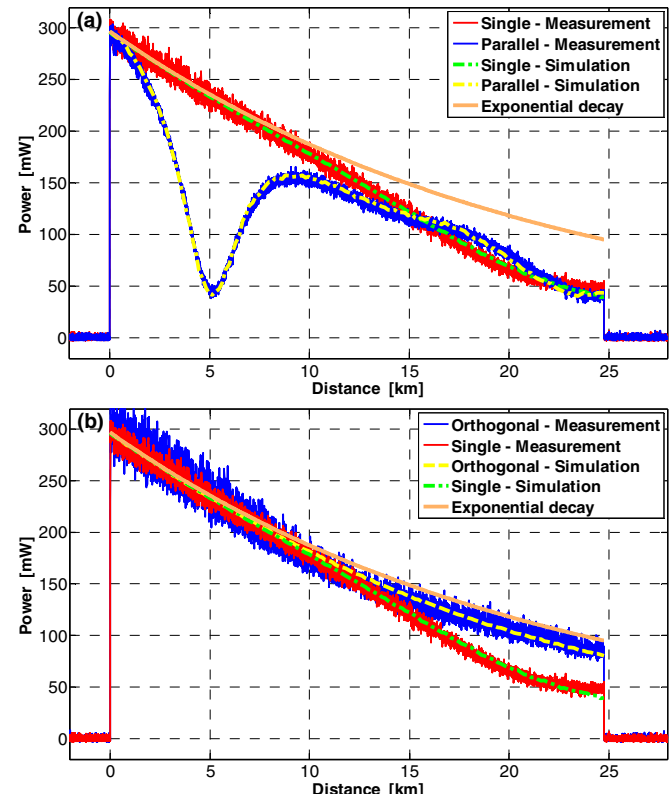

Fig. 3. Measured and simulated power evolution in a $25 \mathrm{~km}$ SMF for an input power of $300 \mathrm{~mW}$ for (a) single pump and double parallel pumps, and (b) single pump and double orthogonal pumps. The system parameters are: $\alpha=0.2 \mathrm{~dB} / \mathrm{km}, \gamma=1.0 \mathrm{~W}^{-1} / \mathrm{km}$ and $\beta_{2}=-23 \mathrm{ps}^{2} / \mathrm{km}$. The frequency spacing is $19.1 \mathrm{GHz}$ and the noise power spectral density is $-109 \mathrm{dBm} / \mathrm{Hz}$.

\section{CONCLUSIONS}

The effect of scalar and vector modulation instabilities on the response of Brillouin distributed sensors has been theoretically and experimentally studied. Results show that the maximum pump power allowed in a BOTDA sensor is defined by Manakov MI when using orthogonally polarized pumps. With such a scheme the sensor can actually outperform a conventional BOTDA as well as schemes using parallel pumps.

\section{REFERENCES}

[1] M. A. Soto and L. Thévenaz, "Modeling and evaluating the performance of Brillouin distributed optical fiber sensors," Opt. Express, vol. 21 no. 25 , pp. 31347-31366, 2013.

[2] S. M. Foaleng and L. Thévenaz, "Impact of Raman scattering and modulation instability on the performances of Brillouin sensors," Proceedings of SPIE, vol. 7753, pp. 77539V, 2011.

[3] M. A. Soto, M. Alem, W. Chen, and L. Thévenaz, "Mitigating modulation instability in Brillouin distributed fibre sensors," Proceedings of SPIE, vol. 8794, pp. 87943J, May 2013

[4] M. Alem, M. A. Soto, and L. Thévenaz, "Modelling the depletion length induced by modulation instability in distributed optical fibre sensors," Proceedings of SPIE, vol. 9157, pp. 91575S, June 2014.

[5] M.A. Soto, et al., "Time and frequency pump-probe multiplexing to enhance the signal response of Brillouin optical time-domain analyzers," Opt. Express, vol. 22, no. 23, pp. 28584 28595, 2014.

[6] J. Urricelqui, et al., "Polarization diversity scheme for BOTDA sensors based on a double orthogonal pump interaction" in J. Lightwave Technol. vol. 33, no. 12, pp. 2633-2638, 2015.

[7] G. P. Agrawal, Nonlinear Fiber Optics, $4^{\text {th }}$ ed. Burlington, MA: Academic Press, 2006.

[8] P. K. A. Wai and C. R. Menyuk, "Polarization mode dispersion, decorrelation, and diffusion in optical fibers with randomly varying birefringence," J. Lightwave Technol., vol. 14, no. 2, pp. 148-157, 1996.

[9] B. Kibler, et al., "Observation of Manakov polarization modulation instability in the normal dispersion regime of randomly birefringent telecom optical fiber" in Advanced Photonics, OSA Technical Digest (online), paper NW2A.2, 2014. 